\title{
Spin gap in doped dimerized chain near half-filling
}

\author{
Xuefan Jiang ${ }^{\mathrm{a}, \mathrm{b}, *}$, G.Y. Guo ${ }^{\mathrm{a}}$ \\ a Department of Physics, National Taiwan University, Taipei 106, Taiwan \\ ${ }^{\mathrm{b}}$ Department of Physics, Changshu College, Changshu 215500, Jiangsu, China \\ Received 11 January 2003; accepted 2 April 2003
}

\begin{abstract}
By applying a variational approximation to the bosonized Hamiltonian, the spin gap in a doped spontaneously dimerized spin chain is investigated analytically based on the $t J J^{\prime}$ model in one dimension in the region of small hole doping and small- $J$ limit. It is found that the spin gap is easily destroyed by hole doping. There is a critical value of the hole density at which the system undergoes a continuous crossover from a frustrated quantum liquid with a spin gap to a Tomonaga-Luttinger liquid without a spin gap. Our results are consistent with that from the exact diagonalization method and can also explain some experimental behaviors in the high- $T_{\mathrm{c}}$ materials.
\end{abstract}

(C) 2003 Elsevier Science B.V. All rights reserved.

PACS: 75.10.Jm; 74.72.- h; 74.20.Mn

Keywords: Spin gap; Doping; Dimerization

The discovery of quasi-one-dimensional (1D) cuprates has stimulated the study of 1D strongly correlated electron systems. Although high- $T_{\mathrm{C}}$ cuprates are at least two-dimensional (2D) systems, it is also interesting to investigate the $1 \mathrm{D}$ model. Since it is relatively easy to explore, 2D strongly correlated systems could share some properties of the $1 \mathrm{D}$ case [1]. On the other hand, frustration of exchange interactions in spin and electron systems may bring exotic ground state that cannot be understood from the classical point of view. Among them, a 1D antiferromagnetic Heisenberg spin system with first- and secondneighbor exchange interactions is known to have a

\footnotetext{
*Corresponding author. Tel.: 0086253596273; fax: 008651252773486.

E-mail address: jxfjxf@jlonline.com (X. Jiang).
}

dimer ground state and to have a spin excitation gap [2-8]. Therefore, an interesting question is how the dimer character of the ground state and the spin gap changes when holes are doped. The effects of frustration in spin was considered to be a candidate causing a spin gap in the itinerant electron systems by the numerical diagonalization where a few holes are doped in the half-filled band [5].

The $t J$ model is one of the simplest models for studying strongly correlated electron systems and is a most important problem for high- $T_{\mathrm{C}}$ superconductivity. Interacting $1 \mathrm{D}$ electron systems generally behave as Tomonaga-Luttinger (TL) liquids $[9,10]$ in which the correlation functions have power-law decays with exponents that depend on the interaction strength. Using exact diagonalization method, the phase diagram for the 
1D models can be determined by the calculation of one correlation exponent as a function of electron density $n$ and the magnitude of the interaction [11]. Similar behavior was found for the entire region $0 \leqslant J / t \lesssim 1$, where the values of the correlation exponents are similar to those found in one-band Hubbard model [12] in the region $U / 4 t \gtrsim 1$. Considering a frustrating next-nearest-neighbor exchange interaction, $J^{\prime}$, in the $t J$ model, a spin gap can be produced near half-filling, i.e. $n \lesssim 1$ [13]. It is found [13] that the magnitude of the spin gap reduces with hole doping. The persistence of the spin gap at finite values of the doping leads to a quantum liquid with enhanced charge-densitywave (CDW) and singlet superconducting correlations. The evidence for a spin gap opening up above $T_{\mathrm{C}}$ in the low-doping compound $\mathrm{YBa}_{2} \mathrm{Cu}_{3} \mathrm{O}_{6.69}$ was shown by neutron experiments [14]. Quite recently, Seidel et al. found that the spin- $\frac{1}{2}$ chains in layered compound $\mathrm{TiOCl}$ undergoes a transition into a spin-Peierls state at low temperatures [15]. Making this material an attractive candidate for hole doping, they argued [16] that if the singlet pairing forces, which drive the undoped system into a dimerized state, are still effective after a small amount of holes has been doped into the system, then, mobile singlet pairs of electrons may form a condensate and give rise to an unconventional form of superconductivity [16]. These typical physical properties may be addressed by studying a dimerized spin chain described by the $t J J^{\prime}$ model in $1 \mathrm{D}$. When holes are introduced the correlation functions of systems are controlled by a single critical exponent related to the charge compressibility. Thus, the phase diagram of this model was pointed out by renormalization group analysis [17] or by exact diagonalization of lattices for finite hole densities $[13,18]$. More recently, an analytic method was proposed to discuss the phase diagram in the region of small doping hole density, $\delta=1-n$, and of small exchange couplings $J$, $J^{\prime} \ll t$ based on Luttinger liquid theory [16]. The spin gap phase appears near the half-filling. In contrast to the case of large- $J$ model, the holes are highly delocalized in small- $J$ limit. Here, we will investigate analytically the spin gap in doped spontaneously dimerized spin chains near halffilling in the small- $J$ limit.
The Hamiltonian of the $t J J^{\prime}$ model reads

$$
\begin{aligned}
H= & -t P \sum_{j}\left(C_{j, \sigma}^{+} C_{j+1, \sigma}+\text { h.c. }\right) P \\
& +J \sum_{j}\left(\mathbf{S}_{j} \mathbf{S}_{j+1}-\frac{1}{4} n_{i} n_{i+1}\right) \\
& +J^{\prime} \sum_{j}\left(\mathbf{S}_{j} \mathbf{S}_{j+2}-\frac{1}{4} n_{j} n_{j+2}\right),
\end{aligned}
$$

where $C_{i, \sigma}^{+}\left(C_{i, \sigma}\right)$ is the creation (annihilation) operator of an electron with spin $\sigma(\uparrow$ or $\downarrow)$ at site $i, \mathbf{S}_{i}$ are spin- $\frac{1}{2}$ operators and $P$ is the projection operator to the state space where doubly occupied sites are prohibited. We also introduce a parameter $\alpha$ for the strength of the frustration given by $\alpha=J^{\prime} / J$. At just half-filling $(n=1)$, the Hamiltonian (1) reduces to an $S=\frac{1}{2}$ frustrated Heisenberg spin chain. In this case, the ground state at $\alpha=\frac{1}{2}$ is the two-fold degenerate with a spin gap [2,4], and a fluid-dimer transition occurs at $\alpha_{c}=0.2411$ [8]. When $\alpha>\alpha_{c}$ the gap spontaneously opened. A rigorous calculation showed that the lowest excited state has an energy gap equal to $0.25 \mathrm{~J}$ at $\alpha=\frac{1}{2}$ [4]. Upon doping of holes, the system may become metallic, and the spin gap is reduced but persists for the finite doping [5]. It is necessary to distinguish the cases $\alpha<\alpha_{c}$ and $\alpha>\alpha_{c}$. At $\alpha>\alpha_{c}$, a spin gap persists at finite values of $\delta$ and overlaps the pairing region even at small $J$ [13]. There are triplet excitations with gap and singlet pairing correlations. At $\alpha<\alpha_{c}$, however, the phase diagram shows a spin-gapped singlet superconducting region only at large $J[9,18]$. In the following, we focus on the triplet excitations in the regime $\alpha>\alpha_{c}$, which do not deal with the pairing correlations which have been treated by using TL liquid arguments [18]. Recently, Ogata et al. decomposed wave functions of the $t J J^{\prime}$ model into a spinless fermion part and a squeezed spin part in the small- $J$ limit for an arbitrary hole density [13]. They claimed that the squeezed spin part is described by a $J J^{\prime}$ model where exchange energies $J$ and $J^{\prime}$ are modified by the hole density, and that the spin gap survives for small doping. We quote the effective spin Hamiltonian for the squeezed 
spins as follows:

$H_{\sigma}=J_{\mathrm{e}} \sum_{j=1}\left(\mathbf{S}_{j} \mathbf{S}_{j+1}+\alpha_{\mathrm{e}} \mathbf{S}_{j} \mathbf{S}_{j+2}\right)$.

In the small doping regime, the effective couplings are found to be linear in $\delta$ as $J_{\mathrm{e}}=J(1-2 \delta+\alpha \delta)$ and $J_{\mathrm{e}}^{\prime}=\alpha_{\mathrm{e}} J_{\mathrm{e}}=J \alpha(1-3 \delta)$, where $\delta$ is the hole density, $\delta=1-n$. The effect of the doped hole is simply to reduce the effective exchange interactions so that the ratio decreases from the bare value $\alpha=J^{\prime} / J$. This means that the frustration of the spin chain is reduced by the doped holes. The magnitude of the gap can be estimated from the results of the spin chain.

Now let us treat the above spin chain within the scheme of the bosonization theory. The gap formation caused by the frustration can be well described by the 1D quantum sine-Gordon (SG) model obtained via the bosonization procedure, the effective Hamiltonian of Eq. (2) is given as in Ref. [19]

$$
\begin{aligned}
H_{\sigma}= & \frac{1}{2 \pi} \int \mathrm{d} x\left[(u K)(\pi \Pi)^{2}+\left(\frac{u}{K}\right)\left(\partial_{x} \phi\right)^{2}\right] \\
& +\frac{g}{a} \int \mathrm{d} x \cos (\sqrt{8} \phi),
\end{aligned}
$$

where $a$ is a short distance cutoff or lattice spacing. $\Pi$ is the momentum density field conjugate to field operator $\phi$, the commutation relation, $[\phi(x)$, $\left.\Pi\left(x^{\prime}\right)\right]=i \delta\left(x-x^{\prime}\right)$, holds. $u$ and $K$ are the spin wave velocity and the Gaussian coupling, respectively, and $g$ stands for the spin umklapp scattering bare amplitude. The coefficients of $u, K$ and $g$ are related to $\alpha_{\mathrm{e}}$ as $u=J_{\mathrm{e}} a \pi^{-1}\left[\pi+3+7 \alpha_{\mathrm{e}}\right]^{1 / 2}[\pi-1-$ $\left.\alpha_{\mathrm{e}}\right]^{1 / 2}, K=2\left[\pi-1-\alpha_{\mathrm{e}}\right]^{1 / 2}\left[\pi+3+7 \alpha_{\mathrm{e}}\right]^{-1 / 2}$, and $g=-J_{\mathrm{e}}\left[1-3 \alpha_{\mathrm{e}}\right] / 2$. For convenience we rewrite here, after making a simple transformation for field operator $\phi \rightarrow \sqrt{\pi K} \phi$, the Hamiltonian in the following form:

$H_{\sigma}=u \int \mathrm{d} x\left[\frac{1}{2}\left(\partial_{x} \phi\right)^{2}+\frac{1}{2} \Pi^{2}-\frac{m_{0}}{\beta^{2}} \cos (\beta \phi)\right]$,

where we define $m_{0}=-2 \pi a^{-2}\left(1-3 \alpha_{\mathrm{e}}\right)(\pi+3+$ $\left.7 \alpha_{\mathrm{e}}\right)^{-1} \quad$ and $\quad \beta^{2}=16 \pi\left(\pi-1-\alpha_{\mathrm{e}}\right)^{1 / 2}(\pi+3+$ $\left.7 \alpha_{\mathrm{e}}\right)^{-1 / 2}$.

It is therefore that the ground-state and the elementary excitation properties of the effective spin Hamiltonian may be derived from the corresponding 1D SG model. It is well known that the 1D SG model is exactly solvable at special values of $\beta$ and the excitation spectrum is well understood [20]. The phase diagram of the 1D SG model was discussed by analytical approximations $[21,22]$. It is found [23] that the $1 \mathrm{D}$ SG model undergoes a phase transition at the critical coupling $\beta^{2}=8 \pi$, from which the critical value of $\alpha_{c}=0.2204$ is obtained. As a phenomenological parameter, however, we will take the accurate critical value of $\alpha_{c}=0.2411$ [8] below. As mentioned above, for $\alpha_{\mathrm{e}}>\alpha_{\mathrm{c}}$ the model Hamiltonian (2) is characterized by an excitation gap, while no gap appears for $\alpha_{\mathrm{e}}<\alpha_{\mathrm{c}}$. This means that since frustration provides principally the mechanism for energy gap formation, following Ref. [24] we adjust two coefficients $m_{0}$ and $\beta$ as $m_{0}=2 \pi a^{-2}\left(\alpha_{\mathrm{e}}-\alpha_{\mathrm{c}}\right)\left(\pi+3+7 \alpha_{\mathrm{e}}\right)^{-1}$ and $\beta^{2}=$ $8 \pi\left[1-\left(\alpha_{\mathrm{e}}-\alpha_{\mathrm{c}}\right)\right]^{1 / 2}\left[1+\left(\alpha_{\mathrm{e}}-\alpha_{\mathrm{c}}\right)\right]^{-1 / 2}$, respectively. It is known that, for the 1D SG model, the main difficulty that occurred in the conventional calculations based on the perturbation theory is the infrared divergency [20]. Recently, based on the pairing boson modes, a variational ground state was constructed to deal with the 1D SG model [21]. This state can be realized with the help of a unitary transformation, and the variational procedure is essentially characterized by one parameter. The pairing ground state implies Bose condensation of the zero momentum mode, and the variational parameter is related to dynamical mass (or energy gap) generation. This method was also expanded to the 1D double-frequency SG model describing a dimerized and frustrated Heisenberg chain at zero temperature [25]. With it the properties of the ground state and the elementary excitation for the 1D spin model can be given analytically. Here, this method is employed to treat Eq. (4). Let us construct a variational ground state by the usual vacuum state $|0\rangle$ as follows:

$\left|G_{\sigma}\right\rangle=\exp \sum_{k}\left(\zeta_{k} / 2\right)\left(b_{k} b_{-k}-b_{k}^{+} b_{-k}^{+}\right)|0\rangle$,

where $\zeta_{k}$ is an undetermined variational parameter related to a self-consistent parameter introduced below. This pair-coupling picture suitably handle the infrared divergence [21]. As a result, the average value of $H_{\sigma}$ in state $\left|G_{\sigma}\right\rangle$ per site can be 
obtained:

$E_{\sigma}=u\left[\sum_{k}(|k| / 2) \cosh 2 \zeta_{k}-\frac{m_{0}}{\beta^{2}} \xi\right]$,

with $\xi=\exp \left[-\beta^{2} / 4 \sum_{k} \mathrm{e}^{-2 \zeta_{k}} /|k|\right]$. The variational parameter, $\zeta_{k}$, is determined by $\partial E_{\sigma} / \partial \zeta_{k}=0$, yielding $\zeta_{k}=\frac{1}{4} \ln \left(1+m_{0} \xi / k^{2}\right)$. Substituting $\zeta_{k}$ into the definition of the parameter $\xi$, $\xi$ is given selfconsistently by

$\xi=\left\{\frac{m_{0} \xi}{\left[1+\left(1+m_{0} \xi\right)^{1 / 2}\right]^{2}}\right\}^{\beta^{2} / 8 \pi}$.

The single-particle excited state takes the form as $\left|\psi_{\sigma}(k)\right\rangle=b_{k}^{+}\left|G_{\sigma}\right\rangle$. We can also evaluate the single-particle excitation spectrum as

$\omega\left(k, \alpha_{\mathrm{e}}\right)=\left(u^{2} k^{2}+\Delta^{2}\right)^{1 / 2}$,

where $\Delta=u \sqrt{m_{0} \xi}$ stands for the spin gap of the singlet-triplet excitation. We have taken $a=1$. The appearance of a gap in the spectrum marks a dynamical mass generation from the free-boson model. When $\xi$ tends to zero, the gap (or mass) vanishes. It is found $[21,22]$ that for $\beta^{2}>8 \pi$, there is no nonzero solution of $\xi$ in Eq. (7), indicating gapless behavior; on the other hand, for $\beta^{2}<8 \pi$, there is a nonzero solution of $\xi$ and so the gap appears.

Carrying out the integral in Eq. (6) for the momentum, we get the energy of the spin part per site to be

$E_{\sigma}=u\left(\frac{1}{4 \pi} \sqrt{1+m_{0} \xi}-\frac{m_{0} \xi}{\beta^{2}}\right)$.

Adding to the energy of the charge part per site, $E_{\rho}=-2 t / \pi \sin (\delta \pi)$ [13], the ground-state energy of the system per site, $E_{0}=E_{\rho}+E_{\sigma}$, can be obtained.

Fig. 1 shows the reduced spin gap $\Delta / J$ as a functions of the hole density, $\delta$, for fixed frustration value. The frustration causes the increase of the spin gap seen from Fig. 1. Compared with the case of $\alpha=0.4$, the hole density dependence of the spin gap for $\alpha=0.5$ is shown as the behavior of an obvious decrease with increasing $\delta$, indicating noticeable interplay of $\alpha$ and $\delta$. From Ogate et al.'s theory [13], the effect of the doped holes is simply to reduce the effective exchange

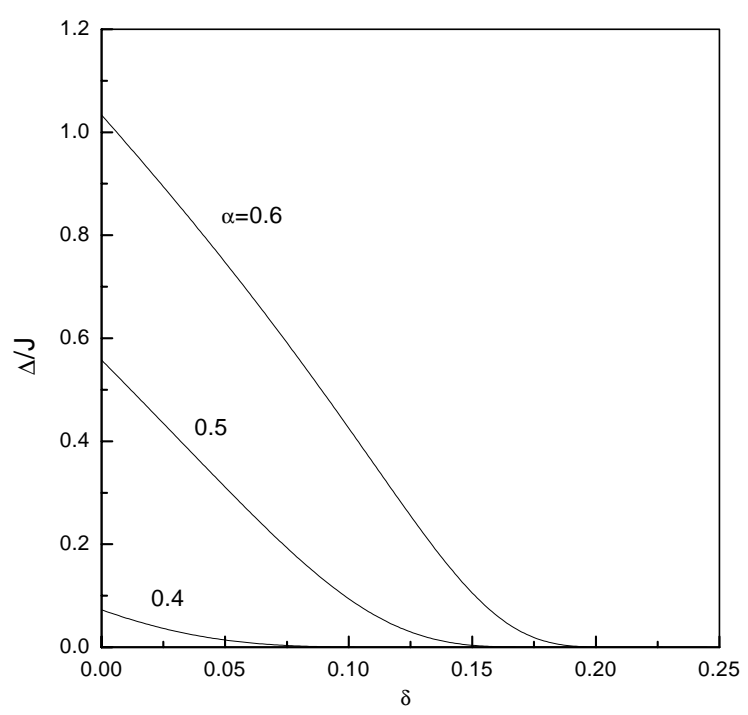

Fig. 1. Spin gap as a function of hole doping density in the small-J limit for fixed frustration.

interactions so that the ratio $\alpha_{\mathrm{e}}$ decreases from the bare value $\alpha$. This means that the frustration of the spin chain is reduced by the doped holes, resulting in the decrease of the spin gap. Fig. 1 shows that the spin gap is easily destroyed by the doping of holes in the spin chain. The magnitude of the spin gap decreases rapidly with increasing hole density and becomes very small at the lower hole density.

Comparing $\alpha_{\mathrm{e}}$ with the critical frustration $\alpha_{\mathrm{c}}=$ 0.2411 , i.e. $\alpha_{e}=\alpha_{c}$, we can obtain the critical hole density $\delta_{\mathrm{c}}$ as a function of the bare frustration $\alpha$ where the spin gap vanishes. When $\alpha$ increases from $\alpha_{\mathrm{c}}$, corresponding $\delta_{\mathrm{c}}$ increases from zero, such that for $\alpha=0.4$ and $0.5, \delta_{\mathrm{c}}=0.195$ and 0.227 , respectively. Analyzing the solutions of the parameter $\xi$ in the self-consistent Eq. (7), we find that $\xi$ decreases continuously from a finite value to be zero as $\delta$ increases starting from zero. It follows, from the definition of the spin gap, that the magnitude of the spin gap decreases and becomes continuously zero when the hole density increases from zero to a critical value $\delta_{\mathrm{c}}$. This behavior indicates that the transition between the gap and the gapless phases is continuous in the small- $J$ limit. It contrasts with the result in the finite- $J$ model by the numerical diagonalization [5], in 
which a bound state among holes and unpaired spins in the spin excited state causes a discontinuity for the spin gap as a function of the electron density. Owing to the existence of the bound state, the first spin excited state cannot be decomposed into a spinless fermion part and a squeezed spin part for finite $J$ model.

The typical physical behaviors obtained are also consistent with that of the numerical method [13]. Our results show a crossover from a frustrated quantum liquid with a spin gap to a TL liquid without a spin gap with increasing doping. Notice that in the high- $T_{\mathrm{c}}$ materials, such as $\mathrm{YBa}_{2} \mathrm{Cu}_{3} \mathrm{O}_{6.69}$ [14], there is an experimental progression with increasing doping from antiferromagnetic long-range order to a quantum liquid with a spin gap above $T_{\mathrm{c}}$ to a quantum liquid which is close to a usual Fermi liquid. Obviously, our results can explain qualitatively the latter type of crossover in the experimental progression mentioned above. We conclude that doping reduces frustration and causes a transition to a TL liquid without a spin gap. Quantitatively, however, our value of $\Delta / J$ is equal to 0.55 at $\delta=0$ and $\alpha=0.5$, which is somewhat twice larger than the exact value of 0.25 . This difference may stem from the fact that we have neglected the renormalization of the parameters in transforming the lattice model Eq. (2) into the continuum model Eq. (3). The other reason may be that a suitable momentum cutoff factor should be selected, here we set $1 / a$ and $a=1$.

In summary, we have presented a method to investigate analytically the spin gap in doped dimerized spin chain described by the 1D $t J J^{\prime}$ model in the region of small doping density and small- $J$ limit. In this case the spin-Hamiltonian is reduced to an effective Hamiltonian defined on the squeezed lattice. The hole doping reduces effective frustration, leading to the decrease of the spin gap. The spin gap is easily destroyed by hole doping. There is a critical value of the hole density at which the system undergoes a crossover from a frustrated quantum liquid with a spin gap to a TL liquid without a spin gap. Our results are consistent with those from the exact diagonalization method and can explain some experimental behaviors in the high- $T_{\mathrm{c}}$ materials.

\section{Acknowledgements}

The authors are grateful to the support from the NSC of Taiwan under Grant No. NSC91-2816-M002-0009-6. X.F.S. was supported in part by the Natural Science Foundation of Jiangsu Department of Education, China.

\section{References}

[1] P.W. Anderson, Phys. Rev. Lett. 64 (1990) 2831.

[2] C.K. Majumdar, D.K. Ghosh, J. Math. Phys. 10 (1969) 1399

C.K. Majumdar, J. Phys. C 3 (1970) 911.

[3] I. Affleck, T. Kennedy, E.H. Lieb, H. Tasaki, Commun. Math. Phys. 115 (1988) 477.

[4] B.S. Shastry, B. Sutheland, Phys. Rev. Lett. 47 (1981) 964.

[5] K. Takano, K. Sano, Phys. Rev. B 48 (1993) 9831; K. Sano, K. Takano, J. Phys. Soc. Japan 62 (1993) 3809.

[6] F.D.M. Haldane, Phys. Rev. B 25 (1982) 4925; F.D.M. Haldane, Phys. Rev. B 26 (1982) 5257.

[7] K. Kuboki, H. Fukuyama, J. Phys. Soc. Japan 56 (1987) 3126.

[8] K. Okamoto, K. Nomura, Phys. Lett. A 169 (1992) 433.

[9] J. Solyom, Adv. Phys. 28 (1979) 201.

[10] F.D.M. Haldane, Phys. Rev. Lett. 45 (1980) 1358.

[11] M. Ogata, M.U. Luchini, S. Sorella, F.F. Assaad, Phys. Rev. Lett. 66 (1991) 2388.

[12] H.J. Schulz, Phys. Rev. Lett. 64 (1990) 2831.

[13] M. Ogata, M.U. Luchini, T.M. Rice, Phys. Rev. B 44 (1991) 12083.

[14] J. Rossat-Mignod, L.P. Regnault, C. Vettier, P. Burlet, J.Y. Henry, G. Lapertot, Physica B 169 (1991) 58.

[15] A. Seidel, C.A. Marianetti, F.C. Chou, G. Ceder, P.A. Lee, cond-mat/0206374.

[16] A. Seidel, P.A. Lee, cond-mat/0212199.

[17] M. Nakamura, K. Nomura, A. Kitazawa, Phys. Rev. Lett. 79 (1997) 3214.

[18] M. Nakamura, J. Phys. Soc. Japan 67 (1998) 717.

[19] K. Kuboki, H. Fukuyama, J. Phys. Soc. Japan 56 (1987) 3126.

[20] A.O. Gogolin, A.A. Nersesyan, A.M. Tsvelik, Bosonization and Strongly Correlated Systems, Cambridge University Press, Cambridge, 1998.

[21] Y.M. Zhang, M.L. Zhou, B.W. Xu, Phys. Rev. B 47 (1994) 898.

[22] G.M. Zhang, H. Chen, X. Wu, Phys. Rev. B 43 (1991) $13,566$.

[23] S. Coleman, Phys. Rev. D 11 (1975) 2088.

[24] G. Bouzerar, A.P. Kampf, G.I. Japaridze, Phys. Rev. B 58 (1998) 3117.

[25] X.F. Jiang, D.Y. Xing, H. Chen, Phys. Rev. 65 (2002) 054421. 